\title{
A perspective of the dynamic structure of the nucleus explored at the single-molecule level
}

\author{
Thomas Dange • Aviva Joseph • David Grünwald
}

Published online: 15 September 2010

(C) The Author(s) 2010. This article is published with open access at Springerlink.com

\begin{abstract}
Cellular life can be described as a dynamic equilibrium of a highly complex network of interacting molecules. For this reason, it is no longer sufficient to "only" know the identity of the participants in a cellular process, but questions such as where, when, and for how long also have to be addressed to understand the mechanism being investigated. Additionally, ensemble measurements may not sufficiently describe individual steps of molecular mobility, spatial-temporal resolution, kinetic parameters, and geographical mapping. It is vital to investigate where individual steps exactly occur to enhance our understanding of the living cell. The nucleus, home too many highly complex multi-order processes, such as replication, transcription, splicing, etc., provides a complicated, heterogeneous landscape. Its dynamics were studied to a new level of detail by fluorescence correlation spectroscopy (FCS). Singlemolecule tracking, while still in its infancy in cell biology, is becoming a more and more attractive method to deduce key elements of this organelle. Here we discuss the potential of tracking single RNAs and proteins in the nucleus. Their dynamics, localization,
\end{abstract}

Thomas Dange and Aviva Joseph contributed equally.

T. Dange $\cdot$ A. Joseph $\cdot$ D. Grünwald $(\bowtie)$

Kavli Institute of NanoScience, Department

of BioNanoScience, Technical University Delft,

Lorentzweg 1,

2628 CJ Delft, The Netherlands

e-mail: d.grunwald@tudelft.nl and interaction rates will be vital to our understanding of cellular life. To demonstrate this, we provide a review of the HIV life cycle, which is an extremely elegant balance of nuclear and cytoplasmic functions and provides an opportunity to study mechanisms deeply integrated within the structure of the nucleus. In summary, we aim to present a specific, dynamic view of nuclear cellular life based on single molecule and FCS data and provide a prospective for the future.

Keywords live cell imaging · single-molecule tracking $\cdot$ nuclear structure $\cdot$ RNA mobility $\cdot$ protein mobility $\cdot$ HIV
Abbreviations
SMT Single-molecule tracking
FRAP Fluorescence recovery after photobleaching
FLIP Fluorescence loss in photobleaching
FCS Fluorescence correlation spectroscopy
STED Stimulated emission depletion
PALM Photo activation localization microscopy
3D-SIM 3D structured illumination microscopy
APD Avalanche photo diode
EMCCD Electron multiplied charged coupled device
poly(A) poly-adenylated RNA
RNA
mRNP Messenger ribo-nucleo-particle
BR2 Balbiani Ring 2
U1 Uridine-rich small nuclear ribo-nucleo-
snRNP particle 


$\begin{array}{ll}\text { NLS } & \text { Nuclear localization sequence } \\ \text { NES } & \text { Nuclear export sequence } \\ \text { RNAPII } & \text { RNA polymerase II } \\ \text { HIV } & \text { Human immunodeficiency virus } \\ \text { RT } & \text { Reverse transcriptase } \\ \text { IN } & \text { Integrase } \\ \text { MA } & \text { Matrix } \\ \text { NC } & \text { Nucleocapsid } \\ \text { Vpr } & \text { Viral protein R } \\ \text { PIC } & \text { HIV preintegration complex } \\ \text { LTR } & \text { Long terminal repeat } \\ \text { TAR } & \text { Trans-activating responsive element in } \\ & \text { the HIV LTR } \\ \text { ARM } & \text { Arginine-rich Motif } \\ \text { RRE } & \text { Rev response element }\end{array}$

\section{Introduction}

Our current picture of cell nuclear function is shifting from the idea of fully assembled molecular complexes towards molecules freely "roaming" in nuclear space (Pombo and Cook 1996; Phair and Misteli 2000; Bentley 2002). Here, transient interactions (proteinprotein, protein-DNA, and protein-RNA) result in a stochastic but directional progression of multi-scale and multi-player processes (Elf et al. 2007; Zenklusen et al. 2008; Darzacq et al. 2009). While this change in paradigm challenges our view of the composition of multi-protein complexes, it also raises questions of how the nuclear space is organized, how the availability of factors is regulated and how interaction sites are found by the "right" interaction partners. While no membrane-bound compartments (such as in the cytoplasm) have been identified, the cell nucleus is functionally and spatially compartmentalized. Specific areas can be labeled, including the nucleolus, PcG bodies, nuclear speckles, Cajal bodies, Gems, cleavage bodies, perinucleolar compartments, the SAM68 nuclear body, PML bodies, etc. (Spector 2001) forming a "patterned" space. As a result, besides crowding effects also found in the cytoplasm, molecules in the nucleus encounter a highly complex, anisotropic landscape (Richter et al. 2007). To probe the dynamic functions of this landscape, an ensemble of technologies (such as FRAP, FLIP, confocal imaging etc.) have been applied to elucidate the dynamics of protein factors and RNA particles (Becker et al. 2002; Braga et al. 2007). Many functional protein factors have been found to vary in their immobile versus mobile states, as well as in their average off rates from potential binding sites (Misteli and Spector 1999; Grunwald et al. 2006b; Gorski et al. 2008; Grunwald et al. 2008a). Similarly, a wide range of mobility distributions exist in RNA studies (Politz et al. 1998; Shav-Tal et al. 2004; Siebrasse et al. 2008). Even nuclear bodies, that appear stationary in imaging, are actually dynamic complexes undergoing a steady exchange of their building blocks (Politz et al. 2006).

Taken together, these data draw a picture of a highly mobile environment with changing fractions of immobilized populations and a continual change in composition. Experimental access to probe this environment and its embedded processes at the molecular scale is limited as: nuclear organization is actively maintained and so can only be studied in living cells; ensemble technologies, being diffraction limited, fail when challenged by molecular interactions on the nanometer length scale; and the high dynamics of these often transient interactions demand collection of data in the milliseconds time scale, limiting the achievable signal-to-noise ratios.

\section{Observing single molecules in the nucleus, a possibility?}

While multiple strategies are employed today to challenge the resolution criteria (Hell 2003; Betzig et al. 2006; Hess et al. 2006; Rust et al. 2006), imaging of single-molecule signals can provide the position of the observed molecule with an accuracy that can overcome the resolution limit by a factor of ten in the living cell, and is in principle capable of providing nanometer precision ${ }^{1}$ (Bobroff 1986; Schmidt et al. 1996; Thompson et al. 2002; Yildiz et

\footnotetext{
${ }^{1}$ Resolution and localization precision are not interchangeable terms. Practically, nonlinearities (on/off states of fluorophores) are used to construct images with high spatial precision in techniques like Photo Activation Localization Microscopy (PALM) while quenching of emission states by stimulated emission (used in Stimulated Emission Depletion, STED) directly increases the resolution of the recorded signal. In PALM, the same routines are used to determine the position of a molecule as in single molecule imaging; however, the latter relies on the isolated observation of one molecule over time to reveal its dynamic property and can easily be adopted to live cell microscopy.
} 
al. 2003). Continuous image acquisition also enables following a single protein or RNA over an extended amount of time utilizing the high localization accuracy for tracking rather than construction of a high resolution image. An immediate advantage is that no synchronization of cellular processes is needed as single-molecule tracking (SMT) is fast enough to observe the sequence of events in real time and synchronization is achieved during data analysis. Finally, by providing superb time and spatial information simultaneously, SMT is perhaps the tool of choice when it comes to investigating the dynamic landscape of molecular interactions in the living cell.

The question however is how fast SMT can be, or in other words, what is the fastest process observable by this technology? SMT has been shown to be fast enough to even follow freely moving molecules in an aqueous solution (Grunwald et al. 2006a). Measurements of nucleocytoplasmic transport have shown definitively that transient interactions in the living cell can be resolved on the nanometer lengthand milliseconds time scales using SMT. In the same way, single protein factors and RNAs in the nucleus have been measured using inert test probes and functional protein factors (Goulian and Simon 2000; Kubitscheck et al. 2005; Grunwald et al. 2006b; Grunwald et al. 2008a). Compared with fluorescence correlation spectroscopy (FCS) or fluorescence recovery after photobleaching (FRAP), SMT provides the means to analyze multiple forms of mobility in heterogeneous environments. Even more, SMT enables observation of the molecular behavior over the whole field of view and not only within either the confocal volume of an FCS spot or the bleached area in FRAP. This makes the technique very valuable for studying intranuclear processes of proteins and RNAs in relation to defined nuclear structures.

However, observation of single molecules in living cells needs to overcome the problem of having too many overlapping signals from the labeled population of molecules. Adjustment of the number of labeled molecules is usually done by: providing small amounts of recombinant fluorescently labeled proteins (e.g., by microinjection), by careful titration of labeled ligand concentrations, by adjusting expression levels of fluorescent reporter molecules (e.g., using leaky promoters), or by signal amplification (e.g., DNA probes, molecular beacons or the MS2 system) (Bertrand et al. 1998; Bratu et al. 2003; Siebrasse et al. 2008). The resulting experimental design is then driven by optimizing the signal-to-noise ratio during data acquisition, usually by a combination of background reduction, signal multiplication on the detector (e.g., APDs or EMCCDs) and very sensitive detection at low read noise conditions. Finally, the observation time needs to be maximized, taking photobleaching rates, desirable signal intensity and applicable amounts of light into consideration.

While many pioneering studies used excitation energies in the range of several kilo Watts per square centimeter, cells and especially nuclear structures and processes are sensitive to high-photon fluxes (Dobrucki et al. 2007; Hoebe et al. 2007; Grunwald et al. 2008b; Heinze et al. 2009). In this context, calibrating the amount of light delivered to the cell is of crucial importance and can be done for broadband light sources as well as for laser based imaging (Grunwald et al. 2008b). Depending on the mobility of the observed molecules and the acquisition speed of the microscope system used, the acquired data is then analyzed based on the tracking of individual signals (see (Grunwald et al. 2008c) for a detailed review). Figure 1 details simple modifications that can be applied to commercially available equipment to improve performance for the detection of single molecules in living cells at appropriate time scales. In short, a laser illumination module (here shown in a customized home-built version, but also commercially available) is combined with a fast switching mechanism and a fast intensity regulator for the laser light and fiber coupled to the microscope. A stable stage with high precision and small drift is used to position samples above the objective lens and to allow for cell manipulation (e.g., microinjection) if needed. Lastly, a sensitive, fast camera, typically an EMCCD, is used for recording the single-molecule signal and is mounted as directly as possible onto the microscope to increase photon transmission. Either sequential imaging or a second detector is used to monitor a cellular reference structure.

To summarize, SMT and FCS provide the means to study single molecules or very small, locally resolved, ensembles of fluorescently labeled molecules in the living cell. In the following, we discuss our current view of the nuclear landscape, dynamical aspects of the nucleus and point out controversial areas that remain. The road to live cell imaging was laid down by confocal microscopy and today's FCS implemen- 


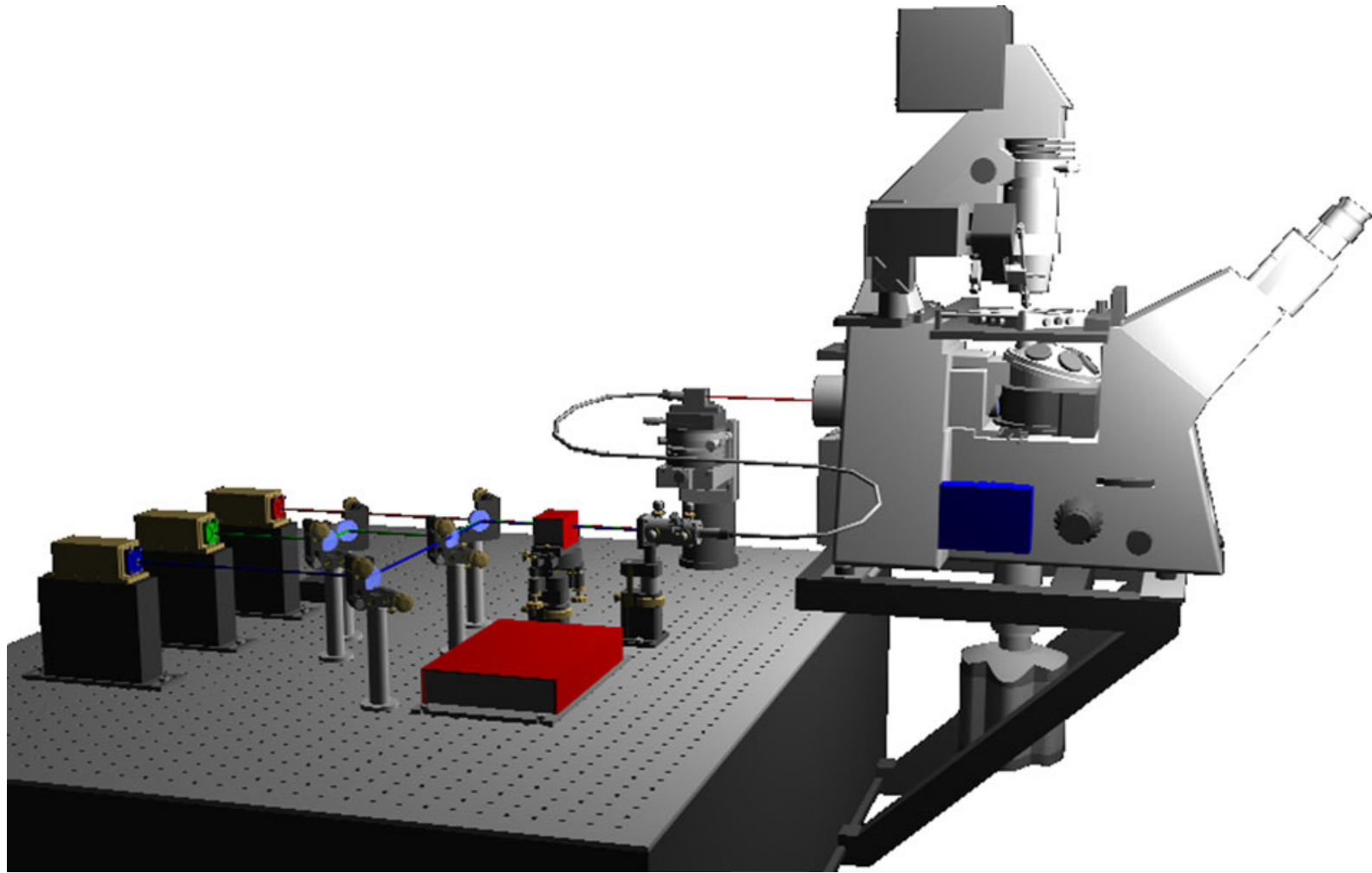

Fig. 1 An SMT microscope setup. A conventional wide-field epifluorescence microscope is custom-modified to optimize single molecule detection in biological samples. Illumination is laser based. Single-mode, low-noise lasers are the preferred choice. The excitation (laser) light is merged using dichromatic beam splitters and mirrors and coupled into an optical monomode fiber to deliver the light to the microscope. An acoustooptical tunable filter is used to attenuate laser power and switch illumination on and off with microsecond resolution. The fiber output can either be delivered as a collimated beam to the excitation tube lens or be imaged via a conjugated image plane into the objective's back focal plane. Emitted fluorescence light, collected by a high numerical aperture objective, is

tations are building upon this technology (Eigen and Rigler 1994).

\section{Nuclear mobility as seen by fluorescence correlation spectroscopy}

The first experiment to provide a mobility profile of the nuclear space used FCS and fluorescein-labeled oligodeoxynucleotides (oligos) to measure intranuclear diffusion coefficients in living cells (Politz et al. 1998). Labeled poly-thymidine oligos, that are passively taken up by living cells, formed hybrids with the poly- separated from the excitation light by an appropriate dichromatic beam splitter, which reflects the three excitation lines into the sample, while the three fluorescence bands are transmitted to the detection system. Notch filters have shown excellent performance in excluding remaining excitation light. The microscope is mounted on a steel scaffold fixed to the optical table to allow easy access to the base port, where an EMCCD for single molecule tracking is mounted directly onto the microscope stand collecting signal in the primary image plane. A second camera, located at a side port, is used to detect reference images. A microinjection system allows delivery of fluorescently labeled probes into a living cell

adenylated tails of RNAs (poly(A)RNAs) in the nucleus, and two major fractions of diffusion rates were observed. Besides the free oligonucleotide diffusion, a slow moving fraction, interpreted as the labeled diffusive RNAs $\left(D=\sim 10 \mu \mathrm{m}^{2} / \mathrm{s}\right)$, was detected. A third minor fraction was also detected with a 10 -fold slower mobility rate (see Table 1). In another experiment, the effect of nuclear compartments on the movement of poly(A)RNAs in the nucleoplasm was compared to poly(A)RNA mobility in speckles (Politz et al. 2006). These values are similar to the mobility of the slower class reported in the first study (Table 1). Interestingly, temperature reduction from $37^{\circ} \mathrm{C}$ to $22^{\circ} \mathrm{C}$ had no 
Table 1 Overview of nuclear diffusion coefficients measured by SMT and FCS

\begin{tabular}{|c|c|c|c|c|}
\hline Reference & Construct & Method & $\begin{array}{l}\text { Diffusion } \\
\text { coefficient }\end{array}$ & Subcompartment \\
\hline (Grunwald et al. 2006b) & U1 snRNP bioactive & SMT & $D=0.5$ to $8 \mu \mathrm{m}^{2} / \mathrm{s}^{\mathrm{a}}$ & Nucleoplasm, speckles \\
\hline (Grunwald et al. 2008a) & Streptavidin-NLS inert & SMT & $D=0.8$ to $5 \mu \mathrm{m}^{2} / \mathrm{s}^{\mathrm{a}}$ & $\begin{array}{l}\text { Nucleoplasm, nucleolus, } \\
\text { heterochromatin }\end{array}$ \\
\hline (Speil and Kubitscheck 2010) & Ovalbumin inert & SMT & $D=0.5$ to $12 \mu \mathrm{m}^{2} / \mathrm{s}^{\mathrm{a}}$ & Nucleoplasm, nucleolus \\
\hline \multirow[t]{5}{*}{ (Bancaud et al. 2009) } & GFP-monomer & FCS & $D_{1}=29 \mu \mathrm{m}^{2} / \mathrm{s}$ & Euchromatin \\
\hline & GFP-monomer & & $D_{1}=87 \mu \mathrm{m}^{2} / \mathrm{s}$ & Aqueous solution \\
\hline & GFP-dimer & & $D_{2}=17 \mu \mathrm{m}^{2} / \mathrm{s}$ & Euchromatin \\
\hline & GFP-dimer & & $D_{2}=55 \mu \mathrm{m}^{2} / \mathrm{s}$ & Aqueous solution \\
\hline & GFP-pentamer & & $D_{3}=7.7 \mu \mathrm{m}^{2} / \mathrm{s}$ & Nucleoplasm \\
\hline (Politz et al. 1998) & Oligo(dT):poly(A) RNA & FCS & $D=1$ to $10 \mu \mathrm{m}^{2} / \mathrm{s}$ & Nucleoplasm \\
\hline \multirow[t]{2}{*}{ (Politz et al. 2006) } & Oligo(dT):poly(A) RNA & FCS & $D_{1}=0.65$ & Speckles \\
\hline & & & $D_{2}=0.7$ & Nucleoplasm \\
\hline \multirow[t]{2}{*}{ (Shav-Tal et al. 2004) } & YFP-MS2 mRNP & FRAP & $D=0.09 \mu \mathrm{m}^{2} / \mathrm{s}$ & Nucleoplasm \\
\hline & & SMT & $D=0.04 \mu \mathrm{m}^{2} / \mathrm{s}$ & Nucleoplasm \\
\hline $\begin{array}{l}\text { (van den Bogaard and } \\
\text { Tyagi 2009) }\end{array}$ & GFP-mRNA-96-mer & SMT & $D=0.033 \mu \mathrm{m}^{2} / \mathrm{s}$ & Nucleus \\
\hline (Siebrasse et al. 2008) & DNA-labeled BR mRNP & SMT & $D=0.24$ to $4.0 \mu \mathrm{m}^{2} / \mathrm{s}$ & Salivary-gland cell nucleus \\
\hline (Mor et al. 2010) & Different sized Dys mRNA & SMT & $D=0.005 \mu \mathrm{m}^{2} / \mathrm{s}$ & Nucleus \\
\hline (Maertens et al. 2005) & HIV-IN & FCS & $D=10.5 \mu \mathrm{m}^{2} / \mathrm{s}$ & Nucleus \\
\hline
\end{tabular}

${ }^{a}$ In these studies, diffusion coefficients were fixed to compare the relative amounts of the different mobility classes in different compartments. For details, see original literature

detectable effect on poly(A)RNA mobility, and was interpreted as meaning that movement between the nucleoplasm and speckles does not require metabolic energy, which is in stark contrast to a previous report (Calapez et al. 2002).

Probing the nuclear space with inert probe molecules, initially described by Grunwald et al. 2008a using SMT, was then used to explore the effects of molecular crowding on diffusion and binding of nuclear proteins in heterochromatin using FCS technology (Bancaud et al. 2009). Volume exclusion, diffusive barriers in more dense nuclear compartments and transient trapping in heterochromatin, as observed by Grunwald et al. 2008a, were confirmed. Using three independent chromatin interacting proteins as active probes, photoactivation experiments were carried out, and in euchromatin, diffusion and binding parameters were well explained by a diffusion limited model. However, when the same probes were tested in heterochromatin, where slower kinetics were expected (dependent on the heterochromatin to euchromatin abundance ratio), an unexpected biphasic mobility was observed that could not be explained by a diffusion reaction model or a random crowding model (Bancaud et al. 2009). Based on these results the authors deduced fractal geometry of chromatin as previously suggested (Wachsmuth et al. 2000).

\section{Single RNA tracking in the nucleus}

Circumventing the small active measurement area and time averaging inherent to FCS, improvements in camera technology, and the development of RNA labeling systems, made imaging of messenger ribonucleoprotein particles (mRNPs) in living cells a possibility. One such labeling system is the MS2 system that consists of two components, i.e., a sequence of MS2 RNA stem-loops introduced into the RNA sequence of interest and a coat protein to which a fluorescent protein is fused. The coat protein binds to the RNA sequence with nanomolar affinity thereby generating a multiplexed signal sufficient for single molecule tracking (Stockley et al. 1995; 
Bertrand et al. 1998; Lago et al. 2001). To detect mRNPs in real time in living mammalian cells, 24 MS2 stem-loops were inserted downstream of a target RNA (Shav-Tal et al. 2004). Movements of individual mRNPs were followed for 100 frames using exposure times of 250 and $333 \mathrm{~ms}$, respectively. The tracking criteria for mRNPs was set to more than eight frames of continuous observation, and the mean diffusion coefficient, in stark contrast to the FCS data cited above, was found to be $0.04 \mu \mathrm{m}^{2} / \mathrm{s}$ at $37^{\circ} \mathrm{C}$. This diffusion was also temperature dependent, as at room temperature the diffusion coefficient was reduced indicating that mRNPs released after transcription moved probabilistically through the nucleoplasm following simple laws of diffusion (Shav-Tal et al. 2004). When very large mRNPs were tracked in the nucleus (Dys-mRNP, ranging from 4.8 to $14 \mathrm{~kb}$ ), an even lower mobility of mRNPs was found $\left(D=\sim 0.005 \mu \mathrm{m}^{2} / \mathrm{s}\right)$ (Mor et al. 2010). Interestingly, in this report, mRNPs were found to travel in chromatin free zones in a "channeled diffusion" manner with the accumulation of mobile particles in distinct chromatin free zones (Mor et al. 2010). While there is evidence of open spaces in the lamin network as revealed by threedimensional structured illumination microscopy (3DSIM) (Schermelleh et al. 2008), it is unclear whether these spaces are indeed channels and if they exist over the entire nucleus as predicted (Mor et al. 2010).

Another labeling approach uses molecular beacons for fluorescent labeling of mRNP (van den Bogaard and Tyagi 2009). A molecular beacon is composed of a stem hybrid that keeps the fluorophore close to a quencher rendering the probe initially non-fluorescent. When the probe hybridizes to a target sequence, the fluorophore separates from the quencher due to a conformational change, and becomes detectable. Sequence specific multiplexing of probes on a target RNA is possible, reaching up to $\sim 100$ probe copies/RNA, resulting in signal intensities of single mRNPs that can be detected as diffraction-limited spots in living cells. In an experiment where imaging was performed at $300 \mathrm{~ms}$ integration time, an average diffusion coefficient of $0.033 \mu \mathrm{m}^{2} / \mathrm{s}$ was found for one half of the observed mRNPs, while the other half displayed a stationary behavior with a calculated diffusion coefficient of $0.0006 \mu \mathrm{m}^{2} / \mathrm{s}$. When the single mRNPs positions were mapped to a chromatin density image the stationary particles were located in high-density chromatin regions. In contrast, the mobile particles were preferably found in low chromatin density areas. ATP depletion increased the number of stalled particles but no strong effect on diffusion was found, again suggesting that mRNP movement is an ATP-independent process (van den Bogaard and Tyagi 2009).

All of these results are $\sim 10-100$-fold lower in their diffusion constants (see Table 1) compared to the FCS based mobility profile (Politz et al. 1998; Politz et al. 2006). In contrast to the above studies, however, when an insect model system (instead of a mammalian cell system) was used, i.e., balbiani ring (BR2) mRNAs (extremely large mRNAs found in the salivary glands of the Chironomus midge), four different mobile fractions, widely varying, were found approaching diffusion coefficients comparable to those found by FCS (Table 1) (Siebrasse et al. 2008). Interestingly, the distribution of diffusion coefficients is better explained as a probability distribution of mobile states that an mRNA molecule can adopt, rather than by the existence of distinct classes of differently mobile mRNAs, a first indication of which was already seen in protein mobility (see Fig. 2 and (Grunwald et al.

Fig. 2 Nuclear protein mobility as determined by SMT. a Displacement-dependent trace analysis of streptavidin in the nucleoplasm. Jump distances were binned into three groups of 0-80 nm, 80-240 nm, and larger than $240 \mathrm{~nm}$ displacement. No difference in observation frequency and decay time is observable between the three binned classes. b The same three classes are used to analyze jump distances of streptavidin molecules inside $\mathrm{MeCP} 2$-induced heterochromatin. A clear decrease in observation frequency for longer jump distances is found reflecting the trap size of this compartment. c Diffusion coefficients are a convenient method to extract a mean mobility value, however, it is a rather "broad" parameter. In cells two or three diffusion coefficients are often used to cover the whole range of jump distances found. Single-molecule observation suggests that individual molecules can adopt different mobile states at different times. Here, we present the mean square displacement of a single streptavidin trace (complete trace black), showing that indeed within one observation interval the molecule changes from a trapped immobile state (blue) to a mobile, diffusive state (cyan) and back to an immobile state (red). d Time projection of the trace analyzed in $\mathbf{c}$ projected onto the reference image from the cell nucleus. The nucleoplasm is colored in green, the pericentric heterochromatin in black. e Overview of individual NLS-Streptavidin-Cy5 traces in a MeCP2-GFP-labeled cell nucleus. Single streptavidin molecules showing different mobility are present in different nuclear subcompartments. The bright areas represent heterochromatin areas. Different colors indicate distinguishable tracks in the nucleoplasm (yellow), in pericentric heterochromatin (green), and the transition between nucleoplasm and heterochromatin (blue). The red-colored track is analyzed in (f). $\mathbf{f}$ The diffusion coefficient was estimated from the mean square displacement versus time of the mobility of the red trace shown in (e) 

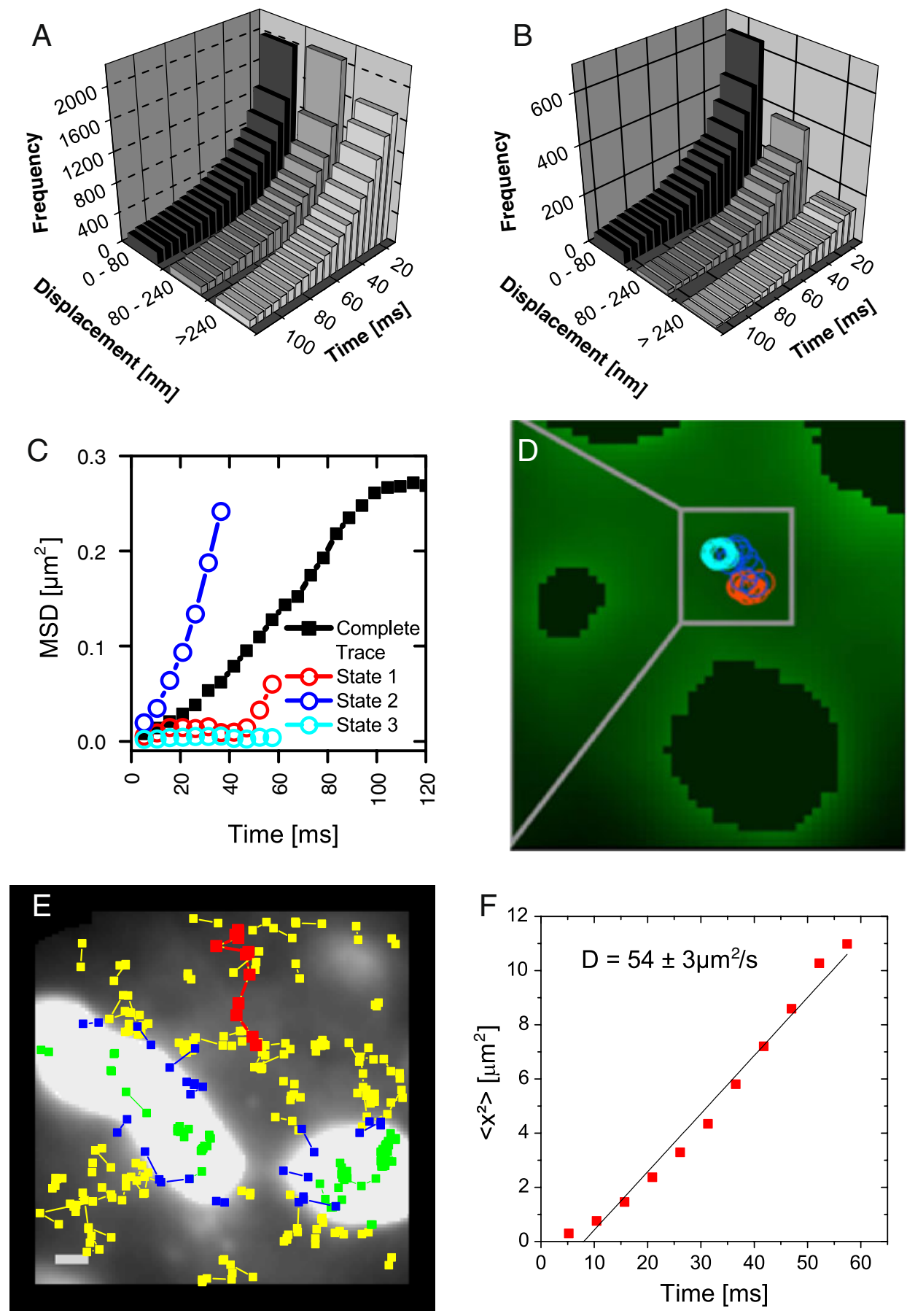
2008a)). Fluorescent labeling here was done either by microinjection of oligos complementary to BR2 mRNA or with a labeled-hrp36 protein that specifically binds BR2 mRNA (Siebrasse et al. 2008).

As there are such dramatic differences between the FCS data and tracking data of one group versus another, it is very clear that controversy exists in this field, which might be explained by clear technical differences in how the experiments were performed.

\section{Single protein tracking in the nucleus}

As we have seen above that RNA tracking is possible, it would be interesting to also track single proteins as proteins are the facilitators of many major cellular processes. One such study looked at the mobility of fluorescently labeled uridine-rich small nuclear ribonucleoproteins (U1 snRNPs), biologically active splicing factors (Grunwald et al. 2006b). GFPlabeled ASF/SF2 was used to mark nuclear speckles allowing direct comparison of U1 snRNP dynamics inside and outside of the nuclear speckles. Using high speed fluorescence microscopy, with frame rates of up to $200 \mathrm{~Hz}$, no significant mobility was found for $\sim 80 \%$ of U1 snRNPs, possibly caused by molecular trapping in nuclear structures as well as immobilization in spliceosomes and post-splicing processes. A continuous range of mobility for U1 snRNPs, ranging from 0.5 to $8 \mu \mathrm{m}^{2} / \mathrm{s}$ was found. The diffusion coefficient of $0.5 \mathrm{\mu m}^{2} / \mathrm{s}$ corresponds to impeded uncomplexed single U1 snRNPs or higher organized spliceosome-complexes. Correspondingly, using FRAP experiments, a three to five fold reduction of the diffusion coefficient of larger molecules in the nuclei was also found (Gorski et al. 2006). From here we conclude that there is not just one kinetic condition for association and dissociation events of biologically active proteins in the nucleus.

How does the large immobile fraction of U1 snRNPs compare, however, to inert proteins? Tagged streptavidin coupled to a nuclear localization sequence (NLS) with a size of about $60 \mathrm{kDa}$, and a second probe, ovalbumin with a size of $45 \mathrm{kDa}$, were tracked in living cell nuclei (Grunwald et al. 2008a; Speil and Kubitscheck 2010). While streptavidin is not translocated by nuclear pores, primarily due to its size, ovalbumin likely passively transports into the nucleus. Using high speed fluorescence imaging at frame rates of up to $200 \mathrm{~Hz}$, the streptavidin experiment succeeded in capturing even single traces of probe molecules and deduced a diffusion rate comparable to that of the inert GFP protein as seen by FCS (see Table 1 and Fig. 2) (Grunwald et al. 2008a; Bancaud et al. 2009). Different nuclear compartments affect the movement of inert proteins differently, but even in the nucleoplasm, two kinetic components (mobile and trapped) were observed, and the mobile fraction is widely spread over a wide range of diffusion coefficients (The data could not be fitted satisfactorily assuming only one or two diffusing components, Table 1). Compared with the nucleoplasm (defined as space neither labeled by MeCP2 or ASF1 exclusion), proteins seemed to become trapped predominantly in pericentric heterochromatin resulting in fewer proteins moving freely (see Fig. 2). This trapping is correlated to the high abundance of chromatin fibers in this area. Even more exciting, and in contrast with the FCS study discussed above (Bancaud et al. 2009), proteins were trapped less frequently in the nucleolus compared to the nucleoplasm. While confocal imaging implies that the nucleolus excludes the test protein, the mobility data suggest minimal trapping in this compartment and combined with no retention of proteins at the compartment interface, exclusion turns out to be a dynamic effect, where mobile proteins leave the nucleolus frequently and fast (Grunwald et al. 2008a; Speil and Kubitscheck 2010). When ovalbumin was used, an inert protein that does not contain an NLS, comparable observations were made demonstrating the limited, if any, impact of the SV40 NLSrelated electric charge on protein mobility in the nucleus.

\section{Imaging HIV in the living cell-a perspective}

An important question that can be addressed using single molecule tracking is the mobility of HIV (Human Immunodeficiency Virus) related factors, their interaction times and regulation of cellular distribution. We first provide a brief summary of the HIV lifecycle, including import and export processes of HIV genetic material through the nuclear membrane, which is a very elegant symphony between cytoplasmic and nuclear viral and host cell factors. Single molecule tracking 
techniques might enable deeper understanding of the spatial-temporal dynamics of these processes thereby not only providing key information on very basic biological processes, but we surmise, also exposing vulnerabilities that could be targeted in the fight against this devastating disease.

\section{The life cycle of HIV}

The HIV virus is an RNA lentivirus with a genome comprising two single-RNA strands containing the full genetic information needed for viral replication in host cells (Luciw et al. 1996; Fields et al. 2007; Levy 2007). Through the process of fusion and endocytosis the virus enters the cell (Fig. 3), by binding its trimeric surface gp120 to a CD4 molecule present on the host cell (reviewed in (Gallo et al. 2003)). This binding induces a conformational change to a specific

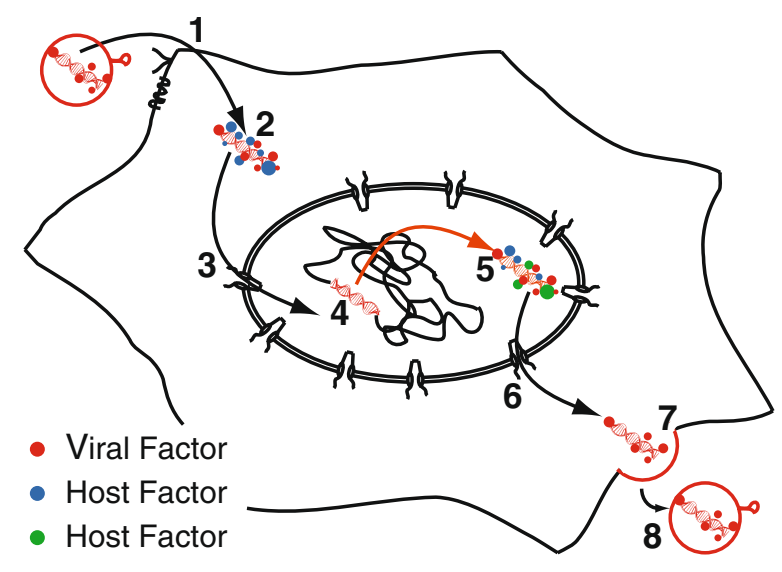

Fig. 3 The life cycle of HIV. 1 The HIV-1 virus binds via its gp120 protein to a host cell's CD4 molecule and a chemokine receptor leading to gp41-mediated viral-host cell membrane fusion. 2 Viral and host cell factors mediate the uncoating, reverse transcription and import of the viral preintegration complex through the nuclear pore (3). 4 Upon arrival in the nucleus, viral genome integration into the host cell's chromosome occurs (again, mediated by viral and host cell factors) and transcription invariably begins. 5 While fully spliced RNAs are exported as usual to the cytoplasm, singly spliced or unspliced RNAs are also exported through the nuclear pore (6) via a REV-mediated mechanism, again assisted by host proteins. 7 Packaging of new virions occurs at the host cell's membrane. 8 In a final fusion event, the newly formed virion is released to continue the infection cycle in a new cell. Each of these various steps could be tracked at the single molecule level. A future promise to enhance our understanding of these steps in more detail is to investigate interactions between selected components by "super-registration" dual color SMT chemokine receptor (predominantly CCR5 or CXCR4 (Berger et al. 1999)), and this in turn induces a conformational change in gp41, another viral surface factor. Gp41 in its altered conformation opens up, thereby "spearing" the lipid bilayer of the target cell. This fusion of the viral and infected cell's membranes results in the release of the HIV viral core into the cytoplasm (Chan et al. 1997; Tan et al. 1997; Weissenhorn et al. 1997; Caffrey et al. 1998). Viral uncoating then takes place (Briones et al. 2010) and the viral reverse transcription complex, comprising viral and host proteins, is assembled and cDNA synthesis begins. The result of reverse transcription is an HIV preintegration complex (PIC), which is then challenged with importing itself into the nucleus. (Lee et al. 2010). It is still unclear how the PIC imports into the nucleus (Dvorin et al. 2002; Bukrinsky 2004; Yamashita and Emerman 2005; Yamashita and Emerman 2006).

Following transport across the nuclear pore complex viral and host proteins again participate in integration of the viral genome into the host cell genome (Greene and Peterlin 2002). While this is primarily mediated by integrase (IN), which binds to the ends of the viral DNA, host proteins are also involved and required, though their precise functions remain unknown (Kalpana et al. 1994; Lee and Craigie 1994; Farnet and Bushman 1997; Li et al. 2000; Suzuki and Craigie 2002; Beitzel and Bushman 2003; Lin and Engelman 2003). LEDGF/p75 also associates with IN and has also been implicated in participating in its nuclear import and/or the integration process (Cherepanov et al. 2003; Maertens et al. 2003). As the HIV provirus can integrate into many different chromosomal locations in the cell it is tempting to explain viral latency (vs. transcriptional activity) as integration into repressed heterochromatin. While this is at least in part true, it is not the whole story (for reviews on HIV viral latency, see (Han et al. 2007; Coiras et al. 2009; Graci et al. 2009)).

In the case of a transcriptionally active integrated provirus, the 5' LTR (long terminal repeat containing promoter elements (Taube et al. 1999)) positions RNA polymerase II (RNAPII) at the site of initiation of transcription and is responsible for the assembly of the pre-initiation complex. While transcription can begin with these minimal components, RNAPII invariably fails to elongate efficiently (Kao et al. 1987). To achieve efficient elongation the viral 
protein Tat is required which associates with host cyclin T1, which in turn recruits host Cdk9. Tat binds the $5^{\prime}$ bulge region of TAR (a 59-nucleotide stem-loop RNA element in the LTR) via its arginine-rich motif and recruitment of P-TEF-b (the complex formed by cyclin $\mathrm{T}$ and $\mathrm{Cdk} 9$ ) results in hyper-phosphorylation of the C-terminal domain of RNA polymerase II, thereby stimulating efficient transcriptional elongation (Zhou et al. 2003).

The integrated provirus, while only $\sim 9 \mathrm{~kb}$ long, then successfully expresses 15 distinct viral proteins, facilitated by an elegant and complex splicing mechanism that involves both complete and incomplete splicing (Frankel and Young 1998). When the mRNA is completely spliced (encoding for Nef, Tat, and Rev) it is rapidly transported into the cytoplasm and transcribed (Cullen 1998). When the mRNA, however, is singly spliced or unspliced, viral transcripts remain in the nucleus and are relatively stable (Luo et al. 1994; Powell et al. 1997). While the export of unspliced (or partially spliced) RNA in eukaryotic cells is usually prohibited, the virus overcomes this block by utilizing the viral protein REV (Custodio et al. 1999). The REV protein binds to an Rev response element (RRE) encoded in the viral RNA sequence and together with recruited host proteins manages to export the unspliced or partially spliced RNA in what is referred to as the REV-RRE-CRM1 export mechanism (reviewed in (Hope 1999)). While the partially (or singly) spliced viral transcripts encode the structural enzymatic accessory proteins, the unspliced RNA species constitute the genome of newly formed progeny virions, and export of these RNAs depend heavily on REV's leucine-rich NES (nuclear-export sequence) as well as on host proteins, predominantly Ran GTPase (Fornerod et al. 1997).

As the viral components needed for new virion formation are assembled in the cytoplasm, shuttling to the plasma membrane (where budding will occur) takes place (reviewed in (Kaplan 2002; Li and Wild 2005; Mazze and Degreve 2006)). This process is highly reliant on the viral Gag polyprotein, but involves, once again, a number of host cell factors, without which the process does not occur (Dussupt et al. 2009). The terminal step in the budding reaction involves a second membrane fusion event for the virus, and this too is an orchestrated effort between the viral Gag polyprotein and host proteins (Kaplan 2002; Li and Wild 2005; Mazze and Degreve 2006).
The virion is then released and free to continue the vicious cycle of infection.

Where to from here? As becomes evident from the wealth of information already available, the past several decades of research have defined a large number of host cell proteins that influence every step of the viral life cycle (Ptak et al. 2008; Fu et al. 2009; Pinney et al. 2009). Initial imaging results on the localization of, for example CD4 and CCR5 on the cellular membrane (Steffens and Hope 2003; Steffens and Hope 2004), RNA distribution in virions (Chen et al. 2009), and the biogenesis of HIV virions at the plasma membrane (Jouvenet et al. 2008) have demonstrated the applicability of microscopy to the HIV field. However, a report that investigated IN mobility within the nucleus by FCS, also demonstrates the current limitations at the nanometer lengthand milliseconds time-scale (Maertens et al. 2005). Clear examples of how SMT could be applied to the study of HIV include analysis of the import and export of viral RNA and proteins, e.g. the PIC complex, the interaction durations and sites for the REV-RRE-CRM1 complex, the distribution of genome integration sites or the interplay of TAT and RNAPII. While the above are good examples of what can be achieved with single-color SMT, investigating the interaction times and complex formation of, for instance HIV-RNA and Rev or IN protein, will require simultaneous multi-color SMT. The problem with this approach is that while in each individual color single molecules will be localized with high precision, the colocalization of these single-molecule positions will remain diffraction limited, hence in the range of more than $200 \mathrm{~nm}$. While single-molecule motors, imaged directly on the cover glass in solution have been double labeled and registered with subdiffraction-limited precision (Churchman et al. 2005), this virtually renders multi-color SMT in the living cell impossible, as in the living cell specific problems with aberrations and chromatic effects inherent to the cellular environment need to be overcome. This problem appears to be overcome by the recent development of a 'super-registration' technique for single molecules with high spatial precision between spectral channels. Using a nuclear pore fluorescent stain it was possible to achieve a $10-\mathrm{nm}$ local registration of nuclear pores and endogenous mRNA, labeled with the MS2 system. "Super-registration" 
allowed following interactions of single mRNAs and pores during export and resolving individual transient steps of the export process and their respective rate constants, which were previously undefined (Grunwald and Singer 2010).

In conclusion, the HIV life cycle demonstrates how important it is to gather a complete picture in time and space of cellular and viral mechanisms that, without microscopy, might not be possible. The horizon for the future should include single-molecule studies on the virus' dynamic equilibrium of processes in living cells that, until now, have been out of reach. Even more, the perspective of investigating individual complexes and molecular interactions in the living cell at the singlemolecule level, e.g., through developments like "superregistration", guides the way towards a quantitative picture of the dynamic equilibrium of cellular life.

Acknowledgements The authors thank Werner Wendler and Ulrich Kubitscheck for their help with Fig. 1 and Volker Buschmann for initial analysis of data presented in Fig. 2.

Open Access This article is distributed under the terms of the Creative Commons Attribution Noncommercial License which permits any noncommercial use, distribution, and reproduction in any medium, provided the original author(s) and source are credited.

\section{References}

Bancaud A, Huet S, Daigle N et al (2009) Molecular crowding affects diffusion and binding of nuclear proteins in heterochromatin and reveals the fractal organization of chromatin. EMBO J 28:3785-3798

Becker M, Baumann C, John S et al (2002) Dynamic behavior of transcription factors on a natural promoter in living cells. EMBO Rep 3(12):1188-1194

Beitzel B, Bushman F (2003) Construction and analysis of cells lacking the HMGA gene family. Nucleic Acids Res 31 (17):5025-5032

Bentley D (2002) The mRNA assembly line: transcription and processing machines in the same factory. Curr Opin Cell Biol 14(3):336-342

Berger EA, Murphy PM, Farber JM (1999) Chemokine receptors as HIV-1 coreceptors: roles in viral entry, tropism, and disease. Annu Rev Immunol 17:657-700

Bertrand E, Chartrand P, Schaefer M et al (1998) Localization of ASH1 mRNA particles in living yeast. Mol Cell 2 (4):437-445

Betzig E, Patterson GH, Sougrat R et al (2006) Imaging intracellular fluorescent proteins at nanometer resolution. Science 313(5793):1642-1645
Bobroff N (1986) Position measurement with a resolution and noise-limited instrument. Rev Sci Instrum 57(6):1152-1157

Braga J, McNally JG, Carmo-Fonseca M (2007) A reactiondiffusion model to study RNA motion by quantitative fluorescence recovery after photobleaching. Biophys J 92 (8):2694-2703

Bratu DP, Cha BJ, Mhlanga MM, Kramer FR, Tyagi S (2003) Visualizing the distribution and transport of mRNAs in living cells. Proc Natl Acad Sci USA 100(23):13308-13313

Briones MS, Dobard CW, Chow SA (2010) Role of human immunodeficiency virus type 1 integrase in uncoating of the viral core. J Virol 84(10):5181-5190

Bukrinsky M (2004) A hard way to the nucleus. Mol Med 10 (1-6):1-5

Caffrey M, Cai M, Kaufman J et al (1998) Three-dimensional solution structure of the $44 \mathrm{kDa}$ ectodomain of SIV gp41. EMBO J 17(16):4572-4584

Calapez A, Pereira HM, Calado A et al (2002) The intranuclear mobility of messenger RNA binding proteins is ATP dependent and temperature sensitive. J Cell Biol 159 (5):795-805

Chan DC, Fass D, Berger JM, Kim PS (1997) Core structure of gp41 from the HIV envelope glycoprotein. Cell 89 (2):263-273

Chen J, Nikolaitchik O, Singh J et al (2009) High efficiency of HIV-1 genomic RNA packaging and heterozygote formation revealed by single virion analysis. Proc Natl Acad Sci USA 106(32):13535-13540

Cherepanov P, Maertens G, Proost P et al (2003) HIV-1 integrase forms stable tetramers and associates with LEDGF/p75 protein in human cells. J Biol Chem 278(1):372-381

Churchman LS, Okten Z, Rock RS, Dawson JF, Spudich JA (2005) Single molecule high-resolution colocalization of $\mathrm{Cy} 3$ and $\mathrm{Cy} 5$ attached to macromolecules measures intramolecular distances through time. Proc Natl Acad Sci USA 102(5):1419-1423

Coiras M, Lopez-Huertas MR, Perez-Olmeda M, Alcami J (2009) Understanding HIV-1 latency provides clues for the eradication of long-term reservoirs. Nat Rev Microbiol 7 (11):798-812

Cullen BR (1998) Retroviruses as model systems for the study of nuclear RNA export pathways. Virology 249(2):203210

Custodio N, Carmo-Fonseca M, Geraghty F et al (1999) Inefficient processing impairs release of RNA from the site of transcription. EMBO J 18(10):2855-2866

Darzacq X, Yao J, Larson DR et al (2009) Imaging transcription in living cells. Annu Rev Biophys 38:173-196

Dobrucki JW, Feret D, Noatynska A (2007) Scattering of exciting light by live cells in fluorescence confocal imaging: phototoxic effects and relevance for FRAP studies. Biophys J 93(5):1778-1786

Dussupt V, Javid MP, Abou-Jaoude G et al (2009) The nucleocapsid region of HIV-1 Gag cooperates with the PTAP and LYPXnL late domains to recruit the cellular machinery necessary for viral budding. PLoS Pathog 5(3): e1000339

Dvorin JD, Bell P, Maul GG et al (2002) Reassessment of the roles of integrase and the central DNA flap in human immunodeficiency virus type 1 nuclear import. J Virol 76 (23):12087-12096 
Eigen M, Rigler R (1994) Sorting single molecules: application to diagnostics and evolutionary biotechnology. Proc Natl Acad Sci USA 91(13):5740-5747

Elf J, Li GW, Xie XS (2007) Probing transcription factor dynamics at the single-molecule level in a living cell. Science 316(5828):1191-1194

Farnet CM, Bushman FD (1997) HIV-1 cDNA integration: requirement of $\mathrm{HMG} \mathrm{I}(\mathrm{Y})$ protein for function of preintegration complexes in vitro. Cell 88(4):483-492

Fields BN, Knipe DM, Howley PM (2007) Fields' virology. Wolters Kluwer Health/Lippincott Williams \& Wilkins, Philadelphia

Fornerod M, Ohno M, Yoshida M, Mattaj IW (1997) CRM1 is an export receptor for leucine-rich nuclear export signals. Cell 90(6):1051-1060

Frankel AD, Young JA (1998) HIV-1: fifteen proteins and an RNA. Annu Rev Biochem 67:1-25

$\mathrm{Fu}$ W, Sanders-Beer BE, Katz KS et al (2009) Human immunodeficiency virus type 1 , human protein interaction database at NCBI. Nucleic Acids Res 37(Database issue):D417-D422

Gallo SA, Finnegan CM, Viard M et al (2003) The HIV Envmediated fusion reaction. Biochim Biophys Acta 1614 (1):36-50

Gorski SA, Dundr M, Misteli T (2006) The road much traveled: trafficking in the cell nucleus. Curr Opin Cell Biol 18 (3):284-290

Gorski SA, Snyder SK, John S, Grummt I, Misteli T (2008) Modulation of RNA polymerase assembly dynamics in transcriptional regulation. Mol Cell 30(4):486-497

Goulian M, Simon SM (2000) Tracking single proteins within cells. Biophys J 79(4):2188-2198

Graci JD, Colacino JM, Peltz SW, Dougherty JP, Gu Z (2009) HIV type-1 latency: targeted induction of proviral reservoirs. Antivir Chem Chemother 19(5):177-187

Greene WC, Peterlin BM (2002) Charting HIV's remarkable voyage through the cell: Basic science as a passport to future therapy. Nat Med 8(7):673-680

Grunwald D, Singer R (2010) In vivo imaging of labelled endogenous $\beta$-actin mRNA during nucleocytoplasmic transport. Nature. doi:10.1038/nature09438

Grunwald D, Hoekstra A, Dange T, Buschmann V, Kubitscheck U (2006a) Direct observation of single protein molecules in aqueous solution. Chemphyschem 7(4):812-815

Grunwald D, Spottke B, Buschmann V, Kubitscheck U (2006b) Intranuclear binding kinetics and mobility of single native U1 snRNP particles in living cells. Mol Biol Cell 17 (12):5017-5027

Grunwald D, Martin RM, Buschmann V et al (2008a) Probing intranuclear environments at the single-molecule level. Biophys J 94(7):2847-2858

Grunwald D, Shenoy SM, Burke S, Singer RH (2008b) Calibrating excitation light fluxes for quantitative light microscopy in cell biology. Nat Protoc 3(11):1809-1814

Grunwald D, Singer RH, Czaplinski K (2008c) Chapter 27 Cell Biology of mRNA Decay. In: Lynne EM, Megerditch K (eds) Methods in enzymology, vol 448. Academic Press, San Diego, pp 553-577

Han Y, Wind-Rotolo M, Yang HC, Siliciano JD, Siliciano RF (2007) Experimental approaches to the study of HIV-1 latency. Nat Rev Microbiol 5(2):95-106
Heinze KG, Costantino S, De Koninck P, Wiseman PW (2009) Beyond photobleaching, laser illumination unbinds fluorescent proteins. J Phys Chem B 113(15):5225-5233

Hell SW (2003) Toward fluorescence nanoscopy. Nat Biotechnol 21(11):1347-1355

Hess ST, Girirajan TP, Mason MD (2006) Ultra-high resolution imaging by fluorescence photoactivation localization microscopy. Biophys J 91(11):4258-4272

Hoebe RA, Van Oven CH, Gadella TW Jr et al (2007) Controlled light-exposure microscopy reduces photobleaching and phototoxicity in fluorescence live-cell imaging. Nat Biotechnol 25(2):249-253

Hope TJ (1999) The ins and outs of HIV Rev. Arch Biochem Biophys 365(2):186-191

Jouvenet N, Bieniasz PD, Simon SM (2008) Imaging the biogenesis of individual HIV-1 virions in live cells. Nature 454(7201):236-240

Kalpana GV, Marmon S, Wang W, Crabtree GR, Goff SP (1994) Binding and stimulation of HIV-1 integrase by a human homolog of yeast transcription factor SNF5. Science 266(5193):2002-2006

Kao SY, Calman AF, Luciw PA, Peterlin BM (1987) Antitermination of transcription within the long terminal repeat of HIV-1 by tat gene product. Nature 330 (6147):489-493

Kaplan AH (2002) Assembly of the HIV-1 core particle. AIDS Rev 4(2):104-111

Kubitscheck U, Grunwald D, Hoekstra A et al (2005) Nuclear transport of single molecules: dwell times at the nuclear pore complex. J Cell Biol 168(2):233-243

Lago H, Parrott AM, Moss T, Stonehouse NJ, Stockley PG (2001) Probing the kinetics of formation of the bacteriophage MS2 translational operator complex: identification of a protein conformer unable to bind RNA. J Mol Biol 305(5):1131-1144

Lee MS, Craigie R (1994) Protection of retroviral DNA from autointegration: involvement of a cellular factor. Proc Natl Acad Sci USA 91(21):9823-9827

Lee K, Ambrose Z, Martin TD et al (2010) Flexible use of nuclear import pathways by HIV-1. Cell Host Microbe 7 (3):221-233

Levy JA (2007) HIV and the pathogenesis of AIDS. ASM, Washington D.C

Li F, Wild C (2005) HIV-1 assembly and budding as targets for drug discovery. Curr Opin Investig Drugs 6(2):148-154

Li L, Yoder K, Hansen MS et al (2000) Retroviral cDNA integration: stimulation by HMG I family proteins. J Virol 74(23):10965-10974

Lin CW, Engelman A (2003) The barrier-to-autointegration factor is a component of functional human immunodeficiency virus type 1 preintegration complexes. J Virol 77 (8):5030-5036

Luciw P, Fields BN, Knipe DM, Howley PM (1996) Human immunodeficiency virus and their replication. In: Field virology. Lippincott-Raven, Philadelphia, pp 1881-1952

Luo Y, Yu H, Peterlin BM (1994) Cellular protein modulates effects of human immunodeficiency virus type 1 Rev. J Virol 68(6):3850-3856

Maertens G, Cherepanov P, Pluymers W et al (2003) LEDGF/ p75 is essential for nuclear and chromosomal targeting of 
HIV-1 integrase in human cells. J Biol Chem 278 (35):33528-33539

Maertens G, Vercammen J, Debyser Z, Engelborghs Y (2005) Measuring protein-protein interactions inside living cells using single color fluorescence correlation spectroscopy. Application to human immunodeficiency virus type 1 integrase and LEDGF/p75. FASEB J 19 (8):1039-1041

Mazze FM, Degreve L (2006) The role of viral and cellular proteins in the budding of human immunodeficiency virus. Acta Virol 50(2):75-85

Misteli T, Spector DL (1999) RNA polymerase II targets premRNA splicing factors to transcription sites in vivo. Mol Cell 3(6):697-705

Mor A, Suliman S, Ben-Yishay R et al (2010) Dynamics of single mRNP nucleocytoplasmic transport and export through the nuclear pore in living cells. Nat Cell Biol 12 (6):543-552

Phair RD, Misteli T (2000) High mobility of proteins in the mammalian cell nucleus. Nature 404(6778):604-609

Pinney JW, Dickerson JE, Fu W et al (2009) HIV-host interactions: a map of viral perturbation of the host system. AIDS 23(5):549-554

Politz JC, Browne ES, Wolf DE, Pederson T (1998) Intranuclear diffusion and hybridization state of oligonucleotides measured by fluorescence correlation spectroscopy in living cells. Proc Natl Acad Sci USA 95 (11):6043-6048

Politz JC, Tuft RA, Prasanth KV et al (2006) Rapid, diffusional shuttling of poly(A) RNA between nuclear speckles and the nucleoplasm. Mol Biol Cell 17(3):1239-1249

Pombo A, Cook PR (1996) The localization of sites containing nascent RNA and splicing factors. Exp Cell Res 229 (2):201-203

Powell DM, Amaral MC, Wu JY, Maniatis T, Greene WC (1997) HIV Rev-dependent binding of SF2/ASF to the Rev response element: possible role in Rev-mediated inhibition of HIV RNA splicing. Proc Natl Acad Sci USA 94(3):973-978

Ptak RG, Fu W, Sanders-Beer BE et al (2008) Cataloguing the HIV type 1 human protein interaction network. AIDS Res Hum Retroviruses 24(12):1497-1502

Richter K, Nessling M, Lichter P (2007) Experimental evidence for the influence of molecular crowding on nuclear architecture. J Cell Sci 120(Pt 9):1673-1680

Rust MJ, Bates M, Zhuang X (2006) Sub-diffraction-limit imaging by stochastic optical reconstruction microscopy (STORM). Nat Methods 3(10):793-795

Schermelleh L, Carlton PM, Haase S et al (2008) Subdiffraction multicolor imaging of the nuclear periphery with 3D structured illumination microscopy. Science 320 (5881):1332-1336

Schmidt T, Schutz GJ, Baumgartner W, Gruber HJ, Schindler H (1996) Imaging of single molecule diffusion. Proc Natl Acad Sci USA 93(7):2926-2929

Shav-Tal Y, Darzacq X, Shenoy SM et al (2004) Dynamics of single mRNPs in nuclei of living cells. Science 304 (5678):1797-1800
Siebrasse JP, Veith R, Dobay A et al (2008) Discontinuous movement of mRNP particles in nucleoplasmic regions devoid of chromatin. Proc Natl Acad Sci 105(51):20291-20296

Spector DL (2001) Nuclear domains. J Cell Sci 114(Pt 16):2891-2893

Speil J, Kubitscheck U (2010) Single ovalbumin molecules exploring nucleoplasm and nucleoli of living cell nuclei. Biochim Biophys Acta 1803(3):396-404

Steffens CM, Hope TJ (2003) Localization of CD4 and CCR5 in living cells. J Virol 77(8):4985-4991

Steffens CM, Hope TJ (2004) Mobility of the human immunodeficiency virus (HIV) receptor CD4 and coreceptor CCR5 in living cells: implications for HIV fusion and entry events. J Virol 78(17):9573-9578

Stockley PG, Stonehouse NJ, Murray JB et al (1995) Probing sequence-specific RNA recognition by the bacteriophage MS2 coat protein. Nucleic Acids Res 23 (13):2512-2518

Suzuki Y, Craigie R (2002) Regulatory mechanisms by which barrier-to-autointegration factor blocks autointegration and stimulates intermolecular integration of Moloney murine leukemia virus preintegration complexes. J Virol 76 (23): 12376-12380

Tan K, Liu J, Wang J, Shen S, Lu M (1997) Atomic structure of a thermostable subdomain of HIV-1 gp41. Proc Natl Acad Sci USA 94(23):12303-12308

Taube R, Fujinaga K, Wimmer J, Barboric M, Peterlin BM (1999) Tat transactivation: a model for the regulation of eukaryotic transcriptional elongation. Virology 264(2):245-253

Thompson RE, Larson DR, Webb WW (2002) Precise nanometer localization analysis for individual fluorescent probes. Biophys J 82(5):2775-2783

van den Bogaard PT, Tyagi S (2009) Using molecular beacons to study dispersal of mRNPs from the gene locus. Methods Mol Biol 464:91-103

Wachsmuth M, Waldeck W, Langowski J (2000) Anomalous diffusion of fluorescent probes inside living cell nuclei investigated by spatially-resolved fluorescence correlation spectroscopy. J Mol Biol 298(4):677-689

Weissenhorn W, Dessen A, Harrison SC, Skehel JJ, Wiley DC (1997) Atomic structure of the ectodomain from HIV-1 gp41. Nature 387(6631):426-430

Yamashita M, Emerman M (2005) The cell cycle independence of HIV infections is not determined by known karyophilic viral elements. PLoS Pathog 1(3):e18

Yamashita M, Emerman M (2006) Retroviral infection of nondividing cells: old and new perspectives. Virology 344 (1):88-93

Yildiz A, Forkey JN, McKinney SA et al (2003) Myosin V walks hand-over-hand: single fluorophore imaging with 1.5-nm localization. Science 300(5628):2061-2065

Zenklusen D, Larson DR, Singer RH (2008) Single-RNA counting reveals alternative modes of gene expression in yeast. Nat Struct Mol Biol 15(12):1263-1271

Zhou M, Deng L, Kashanchi $F$ et al (2003) The Tat/TARdependent phosphorylation of RNA polymerase II C-terminal domain stimulates cotranscriptional capping of HIV-1 mRNA. Proc Natl Acad Sci USA 100(22):12666-12671 